\title{
Selected Aspects of Stand Tests for Prototype Floating Bridge Joints
}

\author{
Wieslaw Krason ${ }^{1, a}{ }^{*}$, Pawel Bogusz ${ }^{2, b}$, Arkadiusz Poplawski ${ }^{3, c}$ and \\ Michal Stankiewicz $z^{3, c}$ \\ ${ }^{1}$ Military University of Technology, Gen. Witolda Urbanowicza Street 2, 00-908 Warsaw, Poland \\ awieslaw.krason@wat.edu.pl, bpawel.bogusz@wat.edu.pl, 'poplawski.arkadiusz@wat.edu.pl, \\ dmichal.stankiewicz@wat.edu.pl
}

Keywords: Floating Cassette, Rod Joint, Strength Tests, Three-Point Bending Test, Force-Displacement Curve, Joint Deformation, DIC Method

\begin{abstract}
The subject of the research is rod joints mounted in a prototype version of a floating module in the form of a metal cassette with adjustable buoyancy. Such joints consist of a movable mandrel and sleeves with a closed cross-section. Experimental stand for a three-point bending test is built on the base of the testing machine. The force-displacement curves are determined for various load cases and the joint working positions. Deformations and displacements of the connector components are also recorded using high-speed cameras. The influence of a blind screw blocking the movement of the mandrel in the connector sleeve on operation in various construction configurations is determined based on the results.
\end{abstract}

\section{Introduction}

Floating bridges belong to the group of special bridges with high mobility. They are most often used by the army to build a temporary water crossing. An important role in the operation of floating bridges is performed by side connectors. They enable fast connection of the same floating segments in a ribbon bridge. Side joints should provide high durability as well as proper operating parameters of the assembled bridge structure. The prototype floating cassettes with adjustable buoyancy [1, 2] are built at the Military University of Technology. Construction works, numerical and experimental tests of the floating crossing system subassemblies are continued [2, 3].

The main connector of the prototype floating cassette shown in Fig. 1a is the subject of the research presented in the paper. The strength joints testing under operating conditions of a complete bridge is difficult (these joints are usually submerged in the water), costly and technically impossible to perform due to the safety of the crew and equipment. Therefore, the research methodology and selected test results of the prototype main connector separated from the bridge cassette - Figure 1b are discussed in the paper. Such joint [4] consist of a movable mandrel and sleeves with a closed cross-section. The connector mandrel locked in the sleeve of one cassette is inserted into the sleeve of the next cassette attached to the ribbon. Such joints can operate as main connectors (mounted vertically on the sides of the cassette) and additional connectors (operating horizontally in the roadway of the cassette) - Fig. 1a. In both of the above cases, these joints work between adjacent cassettes mainly for bending. Therefore, a three-point bending stand of a separated joint on the basis of a testing machine with a sufficiently wide working space is built. An assembly drawing and a separate joint arranged horizontally on the stand are shown in Fig. 1 b, c.

(c) (1) Content from this work may be used under the terms of the Creative Commons Attribution 3.0 license. Any further distribution of this work must maintain attribution to the author(s) and the title of the work, journal citation and DOI. Published under license by Materials Research Forum LLC. 


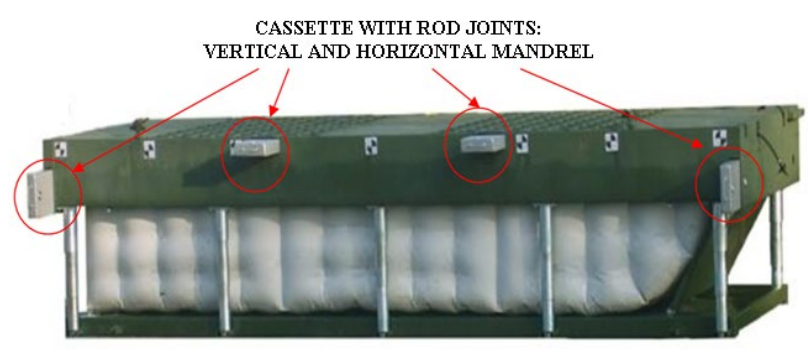

a)

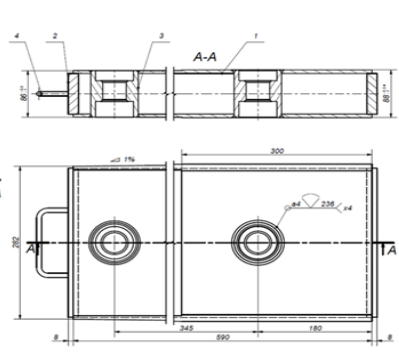

b)

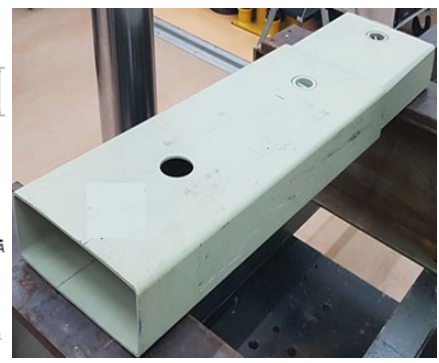

c)

Fig. 1a). View of a cassette with rod joints on the sides (vertical mandrel) and in the cassette roadway (horizontal mandrel), 1b) joint drawing, c) separated joint for stand tests

During the laboratory tests, the force-displacement' curves are determined for the joint in various settings, supports and structural configurations (with or without a mandrel locking by a set blind screw). The maximum displacement values of the joint elements at the stand during the load tests are additionally recorded using a high-speed camera. Displacements of the selected points of the bent joint are also determined along its length and height based on the results obtained by digital image correlation (DIC) methods. The comparison of displacement results will allow for evaluation of flexibility of the subassembly components of the connector stand during testing. The influence of the blind screw locking the movement of the mandrel in the connector sleeve on the operation of these components in various structure configurations of the connector is determined on the basis of the results

\section{Stand for laboratory tests of the joint}

Strength testing stands for the bridge joint are built. A strength machine is used for this purpose. A separated joint for testing has been attached to the machine base. The movable machine punch generated the joint bending force. Fig. 2 shows a diagram of the stands built for three-point bending tests of a joint.

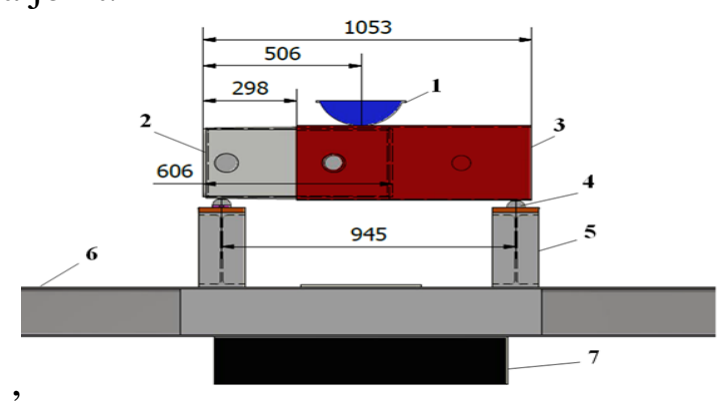

a)

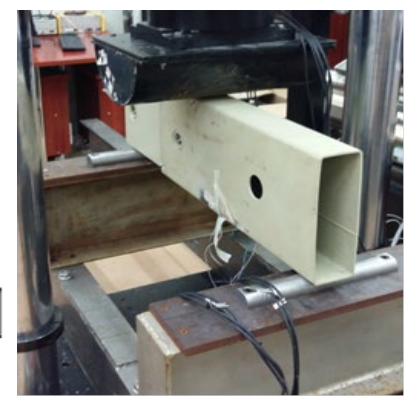

b)

Fig. 2. Structure of the station for experimental investigations of the joint: a) diagram of the three-point bending stand, b) separate rod joint prepared for the three-point bending test

A joint separated for tests is performed based on the design documentation of the cassette. The basic components and the structure of the three-point bending stand for testing of the separated joint on the strength machine are shown in Fig. 2a. The diagram shows the basic components of the three-point testing stand: 1-punch of the testing machine, 2 - mandrel of the tested joint, 3 - sleeve joint, 4 - half-shaft, 5 - moveable base, 6 - I section beam, 7 - base of the testing machine. A stand ready for testing with the joint set in a vertical position is shown in Fig. 2b. An additional equipment (cameras, lighting system and others) used to determine displacements and deformation of subassemblies is shown in Fig. 4a. 


\section{Research results}

Laboratory tests of three-point bending are carried out using the above discussed stand. The rod joint positioned in various settings is bent by a vertical force with different values generated by the punch of the strength testing machine. The 'Force-Displacement P-V' curves are determined for joints in various settings, supports and structure configurations (with or without a mandrel locking by a set blind screw).

Selected results of a three-point bending of the joint placed vertically on the test stand are shown in Fig. 3. Both graphs were obtained when the force changed from $P_{\min }=0$ to the maximum value $\mathrm{P}_{\max }=100 \mathrm{kN}$. The wider hysteresis loop, shown in Figure 3a, refers to the test case of the joint with the blind screw locking the mandrel position in the connector sleeve. The deformation of the joint after the unloading process is noticeable. The value of displacements recorded after unloading to $\mathrm{P}_{\min }=0$ is up to $\mathrm{V}=1 \mathrm{~mm}$. It may result from blocking the mandrel in the sleeve after resetting the nominal clearance due to the limited rotation of the mandrel against the locking blind screw- Fig. $4 \mathrm{~b}$.

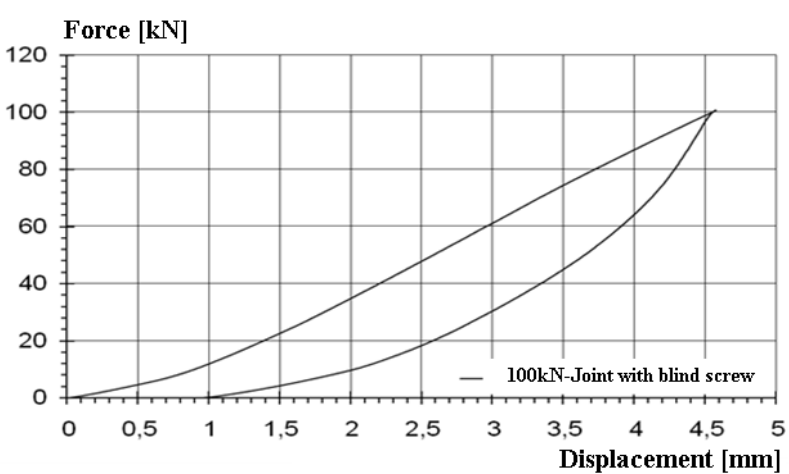

a)

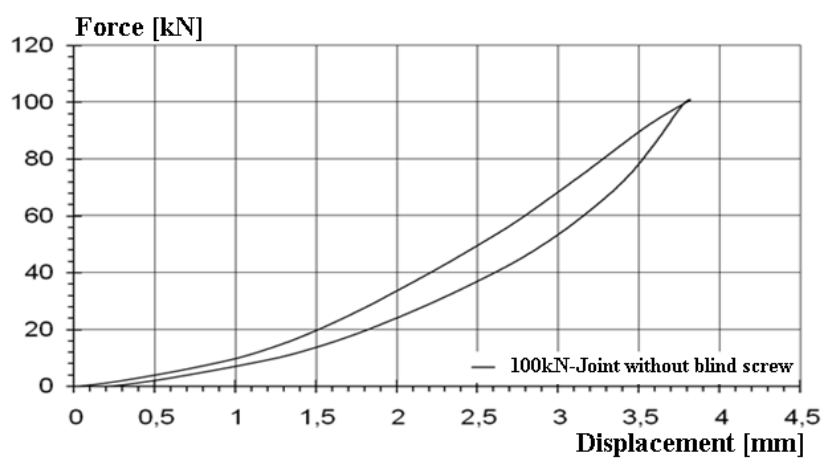

b)

Fig. 3 Graphs of load changes as a function of joint deflection in a three-point bending test with force of $100 \mathrm{kN}$ : a) with a blind screw blocking the position of the mandrel in the connector sleeve, $b$ ) without a set blind screw

The tested joint consists of components that can move relative to each other in a range limited by the initial clearances. These gaps occur due to a difference in nominal dimensions of the mandrel and the connector sleeve. They enable and simplify the connection of the joint components by inserting the mandrel into the sleeve - Fig. 1b, c. A detailed analysis of the joint components displacements towards the horizontal and vertical axis is necessary due to the independent movement of the mandrel and the connector sleeve. Therefore, changes in the displacement values of selected joint elements at the stand during the loading tests are additionally recorded using a high-speed camera - Fig. 4a. Five markers named Point 1-5 (Fig. 4b) are glued on the lateral surface of the mandrel and the sleeve of the joint set vertically. They are used to register the displacement components of the joint subassemblies with cameras, using digital image correlation (DIC) method. The graphs describing changes in the components of the joint displacements during load tests are obtained on the basis of the data recorded with the camera and processed using the Tema software. Selected results of the changes in vertical and horizontal displacements during the three-point bending test of the joint with the maximum force $P_{\max }=100 \mathrm{kN}$ are shown in Fig. 5-8.

The diagrams of the vertical displacements changes recorded by the camera for the subassemblies of the joints with the blind screw locking the position of the mandrel in the sleeve are shown in Fig. 5. Fig. 6 presents the analogous results obtained in the joint tests without the 
set blind screw. The graphs of the horizontal displacements changes recorded by the camera for the joint subassemblies respectively with the blind screw locking the position of the mandrel in the sleeve, and the diagrams of the horizontal displacement changes registered in the joint components during tests without the set blind screw are presented in Fig. 7 and Fig. 8, respectively.

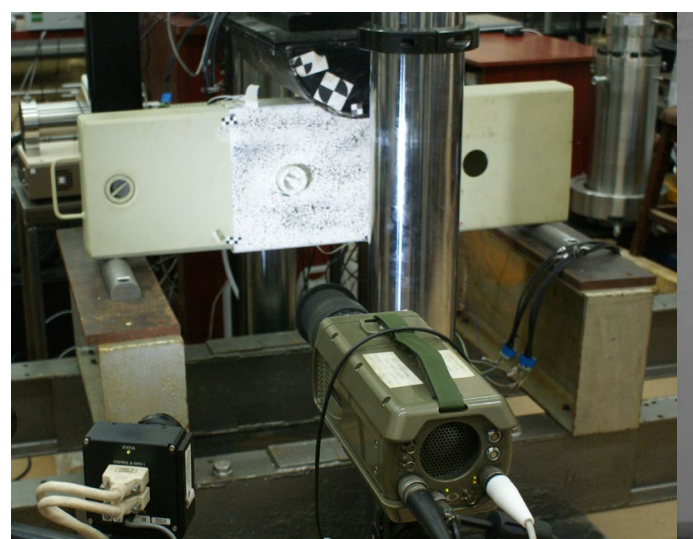

a)

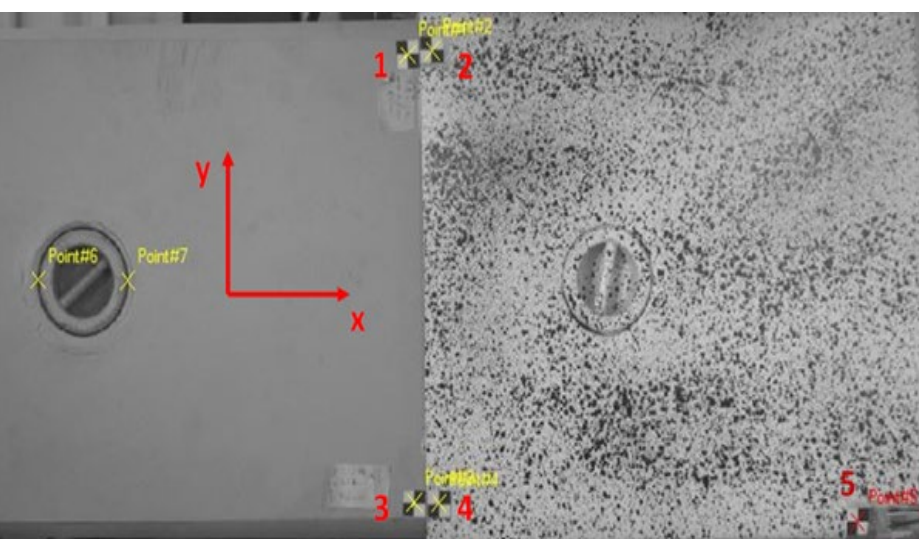

b)

Fig. 4. Stand for registering independent displacements of the joint components with the blind screw; a) an equipment of the test stand with cameras, b) the tested joint with the set blind screw locking the mandrel against the sleeve and markers described with red numbers in the range of 1 - 5

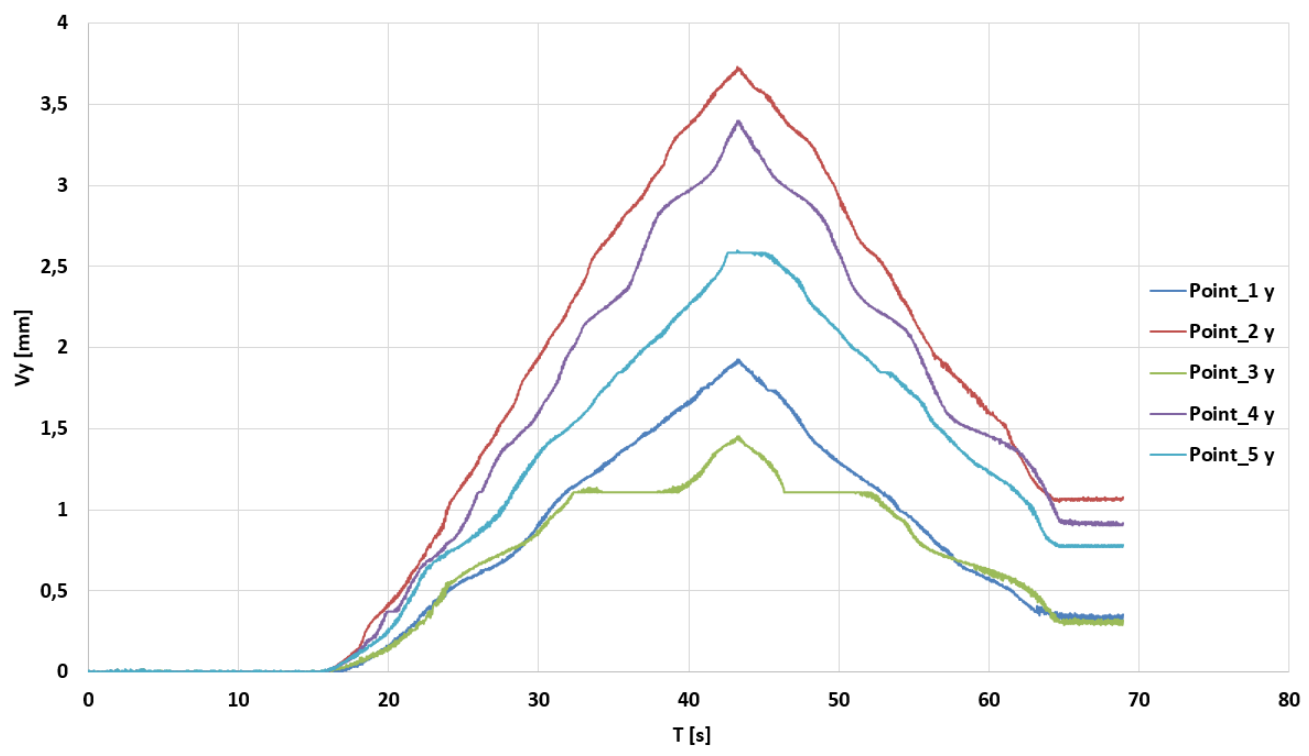

Fig. 5. Graphs of the vertical displacements changes registered for various measuring points (Fig. 4b) of the joint components with the blind screw locking the mandrel with respect to the sleeves

The maximum vertical displacements are recorded in point 2 (Fig. 4b) on the side wall of the joint sleeve in the test variant with the blind screw locking the mandrel. They are $\mathrm{V}_{\mathrm{Y}}=3.7 \mathrm{~mm}$. The maximum horizontal displacement equal to $\mathrm{V}_{\mathrm{X}}=0.7 \mathrm{~mm}$ is recorded in point 5 (Fig. $4 \mathrm{~b}$ ), also on the side wall of the joint sleeve during the test variant with the set blind screw. 


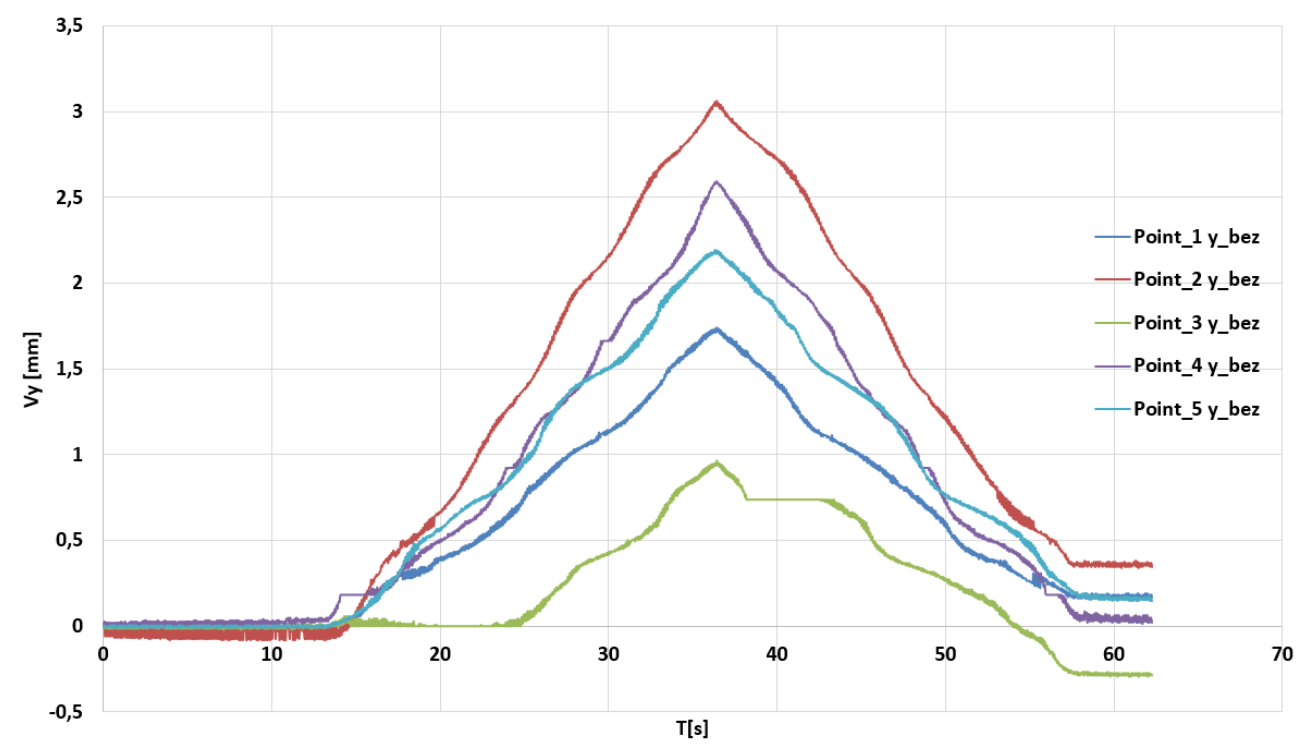

Fig. 6. Graphs of the vertical displacements changes registered for various measuring points

(Fig. $4 b$ ) in the joint components without a set blind screw locking respect to the sleeve

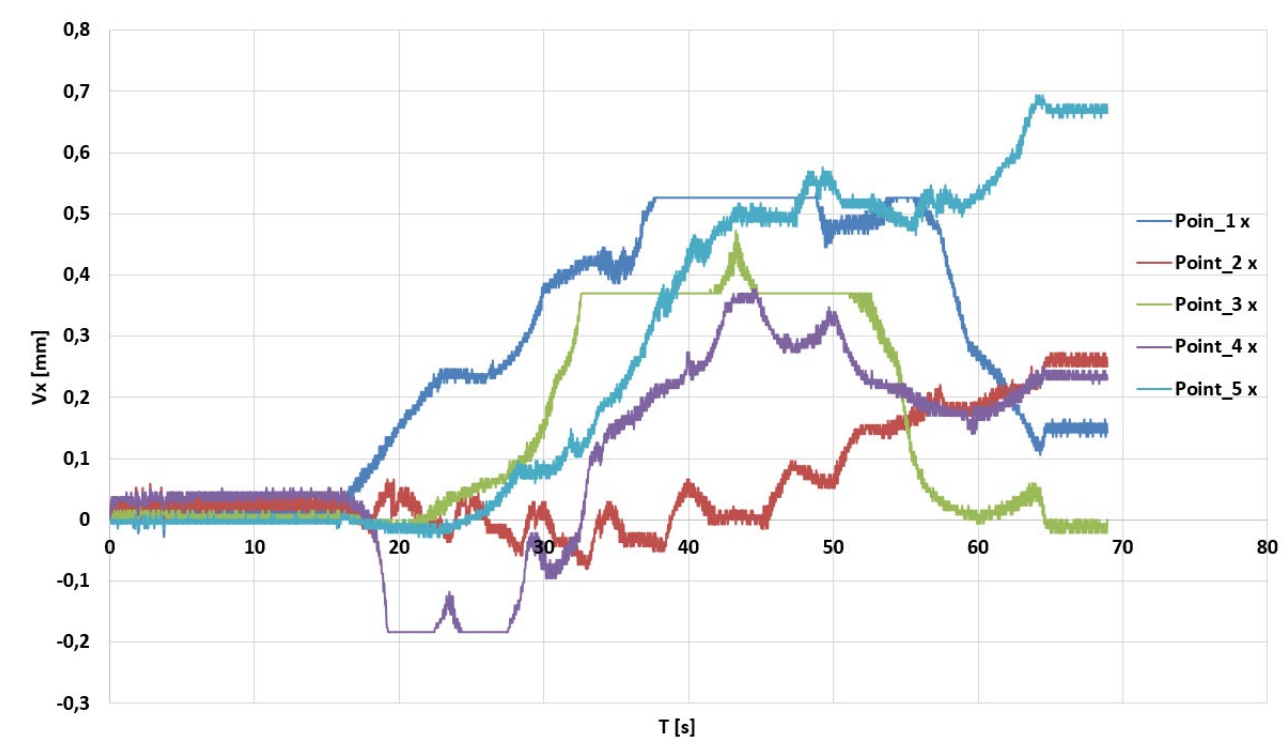

Fig. 7. Graphs of the horizontal displacements changes registered for various measuring points

(Fig. 4b) in the joint components with a set blind screw locking respect to the sleeve

\section{Summary and Conclusions}

The laboratory stand built on the basis of the Satec1200 testing machine is used for a three-point bending test of the separated joint. The load tests of the joint separated from the cassette are developed with the vertical settings of the mandrel (as in the main rod joint of the floating cassette $[3,4]$ ) and various structure configurations of the joint subassemblies. They correspond to the use of a set blind screw between the mandrel and the joint sleeve and the free insertion of the mandrel into a hole of the sleeve without the blind screw locking a mandrel 


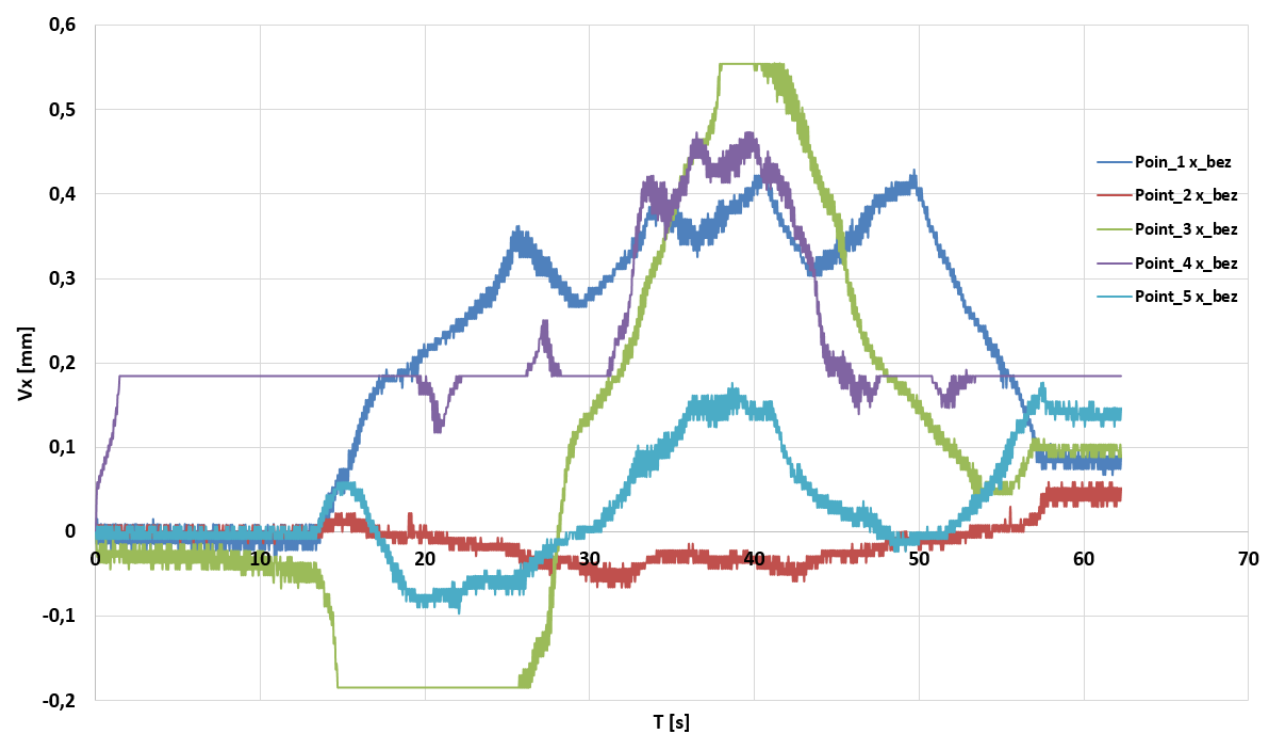

Fig. 8. Graphs of the horizontal displacements changes recorded in various measuring points (Fig. $4 b$ ) of the joint components without the blind screw locking the mandrel with respect to the sleeves

Displacements of the joint components are recorded using the control system of the strength machine ('Force-Displacement' P-V graphs) and with an optical system during the load tests. It is found that larger hysteresis loops appear in P-V curves in the case of the data registered in the joint test variants with the blind screw locking the position of the mandrel in the sleeve.

It is possible to independently track the movement of the joint components along the vertical axis and the horizontal plane of the image with the use of high-speed cameras. Based on the test results, it is also found that the lack of the blind screw does not cause a slide of the mandrel from the joint sleeve during the three-point bending test.

The result of measurements indicates a significant impact of the blind screw locking the mandrel in the joint sleeve on a mechanism of the joint components interaction (mainly the important role of the right size clearances between the mandrel and the sleeve).

\section{Acknowledgement}

The works were carried out as part of the research project PBS 23-937, Military University of Technology, 2016-2018.

\section{References}

[1] Military University of Technology, European Patent, No. 2251255, A sectional pontoon bridge. (2013)

[2] W. Krason, J. Malachowski, Field test and numerical studies of the scissors-AVLB type bridge, Bulletin of The Polish Academy of Sciences, Technical Sciences, Vol. 62, No. 1 (2014) 103-112. https://doi.org/10.2478/bpasts-2014-0012

[3] W. Krason, P. Slawek, Design and pre-testing of a mobile modular floating platform with adjustable displacement, Mechanik nr 11(2017) 1075-1080.

https://doi.org/10.17814/mechanik.2017.11.185

[4] Military University of Technology, Patent Office of the RP, PAT.223689, Set of mechanical locks to connect the floating bridge cassettes and the cassette opening mechanism. (2016) 